\title{
Distributed-memory
} multi-GPU block-sparse tensor contraction for electronic structure

Thomas Herault, Yves Robert, George Bosilca, Robert J. Harrison, Cannada A. Lewis, Edward F. Valeev

RESEARCH 



\title{
inzía
}

\section{Distributed-memory multi-GPU block-sparse tensor contraction for electronic structure}

\author{
Thomas Herault*, Yves Robert ${ }^{\dagger *}$, George Bosilca*, Robert J. \\ Harrison $^{\ddagger}$, Cannada A. Lewis ${ }^{\S}$, Edward F. Valeev $\mathbb{I}$ \\ Project-Team ROMA
}

Research Report n 9353 - June 2020 - 32 pages

\begin{abstract}
Many domains of scientific simulation (chemistry, condensed matter physics, data science) increasingly eschew dense tensors for block-sparse tensors, sometimes with additional structure (recursive hierarchy, rank sparsity, etc.). Distributed-memory parallel computation with block-sparse tensorial data is paramount to minimize the time-tosolution (e.g., to study dynamical problems or for real-time analysis) and to accommodate problems of realistic size that are too large to fit into the host/device memory of a single node equipped with accelerators. Unfortunately, computation with such irregular data structures is a poor match to the dominant imperative, bulk-synchronous parallel programming model. In this paper, we focus on the critical element of block-sparse tensor algebra, namely binary tensor contraction, and report on an efficient and scalable implementation using the task-focused PaRSEC runtime. High performance of the block-sparse tensor contraction on the Summit supercomputer is demonstrated for synthetic data as well as for real data involved in electronic structure simulations of unprecedented size.
\end{abstract}

Key-words: Electronic structure, tensor contraction, block-sparse matrix multiplication, distributed-memory, multi-GPU node, PaRSEC.

\footnotetext{
* Innovative Computing Laboratory, University of Tennessee, Knxoxville, TN, USA

† LIP, École Normale Supérieure de Lyon, CNRS \& Inria, Lyon, France

¥ Stony Brook University \& Brookhaven National Laboratory, Stony Brook, NY, USA

$\S$ Sandia National Laboratories, Livermore, CA, USA

I Department of Chemistry, Virginia Tech, Blacksburg, VA, USA
}

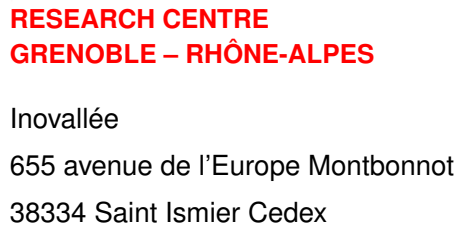




\section{Contraction de tenseur creux par blocs sur plates-formes distribuées équipées de noeuds multi-GPUs}

Résumé : Les tenseurs creux par blocs (block-sparse) sont présents dans de nombreux domaines scienfifiques. Ce rapport étudie la parallélisation d'un noyau de contraction essentiel pour la manipulation de tels tenseurs, qui peut se matérialiser sous forme d'un produit de matrices $C \leftarrow C+A B$, où les trois matrices ont une structure creuse par blocs, où les tuiles de $A$ et $B$ sont de tailles hétérogènes, et où $B$ est carrée de taille $n$, alors que $A$ et $C$ sont rectangulaires de taille $m \times n$ avec $m \ll n$. Nous proposons une implémentation sur la plate-forme Summit à mémoire distribuée, où chaque noeud est équipé de plusieurs GPUs, au sein de l'environnement de tâches $\mathrm{P} a R S E C$. Nous obtenons de bonnes performances pour des problèmes de taille inégalées à ce jour.

Mots-clés : Contraction de tenseur, produit de matrices creuses par blocs, mémoire distribuée, noeud multi-GPU, , $\mathrm{P} a R S E C$ 


\section{Introduction}

The current path to exascale computing relies on an extensive use of accelerators. As of today, the Summit and Sierra systems [31] are the fastest machines on the TOP500 list [48]. Both systems are distributed-memory platforms where each node is equipped with several high performance NVIDIA accelerators. For instance Summit nodes include 6 NVIDIA V100 GPUs, interconnected at the node level by multiple NVLinks. The forthcoming Frontier exascale system [31] is announced with four AMD Radeon GPUs per node. On Summit, more than $97 \%$ of the overall compute performance is on the GPU side. The emerging trend remains consistent across all stateof-the-art platforms equipped with accelerated nodes: these machines draw most of their computing power out of the accelerators; hence, it is crucial, for any efficient and scalable algorithm, to be able to extract the most performance out of the accelerators to achieve high overall efficiency.

The existence of highly capable hardware only translates in application performance if software support exists. The community effort is well on its way to implement dense linear algebra libraries for multi-GPU accelerated nodes. Several on-going projects aim at designing dense linear algebra kernels, not only to achieve high TOP500 performance, but to allow a broad range of applications to benefit from the computing power lying in the accelerators. While most projects are conducted by vendors (Intel, AMD, NVIDIA, Cray), some academic projects, such as SLATE [19,27], are publicly available, and provide efficient CPU or GPU implementations for most traditional dense linear routines. Recently, support for a limited number of operations in a multiple-accelerator setting has been added, with some matrix-size constraints. For instance the current matrix product $C=A \times B$ is limited to problems where the entire $C$ matrix can reside in the memory of the accelerators. A similar academic effort proposes a distributed multi-accelerators prototype for matrix-matrix multiplication without any size restriction within the PaRSEC task-based runtime system [22].

Achieving good performance for dense linear algebra kernels is only a first step to achieving exascale performance for general scientific applications. This can be seen by looking at the performance discrepancies between two of the most widely used benchmarks in HPC, the HPL (High Performance LINPACK) benchmark used in the Top500 list, and HPCG (High Performance Conjugate Gradient) benchmark, more representative of the behavior of a typical scientific application. On Summit, the performance of HPCG is 50 times lower than that of HPL. This is because HPCG involves a communication-bound kernel with sparse fine-grained computational kernels, as opposed to a computation-bound kernel with dense Level3-BLAS routines for HPL.

This paper aims at complementing the insight gained from the HPCG benchmark by exploring another important and widely used computational 
kernel in High Performance Computing. We consider how the binary contraction of block-sparse tensors, a key paradigmatic operation for a variety of physical simulation and data-science domains, can be implemented efficiently on large-scale distributed-memory multi-GPU accelerated platforms. To assess the performance, we consider a mix of synthetic problem setups and contractions taken from actual simulations of electronic structure of molecules. The binary tensor contraction will be mapped, as is typically done, onto the GEneral Matrix Multiplication (GEMM) $C \leftarrow \alpha A B+\beta C$. While the dense matrix multiplication is a formidable, but manageable, challenge on distributed memory heterogeneous platforms for the relevant problem sizes [22], the block-sparse matrix multiplication adds several new challenges. First, the rows and columns of the three matrices are tiled nonuniformly, due to the nonuniform structure of the underlying physical problem. Second, the matrices are block-sparse, with the fill degree greatly varying with the particular simulation from $100 \%$ (for high-precision simulation on compact molecules) to a few percent even for modestly-sized simulations. Third, the aspect ratios of the matrices can vary greatly from 1 (square) to 100s (tall-and-skinny, or short-and-wide); the particular paradigmatic example from the electronic structure domain that we will focus on, involves a large square matrix $B$ and short-and-wide matrices $A$ and $C$, with aspect ratios on the order of 100 . All these characteristics decrease potential data-reuse and arithmetic intensity, and dramatically complicate the design of an efficient algorithm targeting multi-GPU accelerated nodes. The main contribution of this work is the design of a generic and flexible implementation of this block-sparse kernel, and its analysis on a large multi-GPU platform.

The rest of the paper is organized as follows. Section 2 surveys the motivating science application. Section 3 overviews the main design principles of our algorithm. Section 4 discusses the main details of the prototype implementation, which is publicly available [23]. In Section 5, we report preliminary performance results. Section 6 briefly discusses related work. Finally, Section 7 is devoted to concluding remarks and directions for future work.

\section{Motivating Science Application}

Our goal is to deploy the distributed memory block-sparse matrix multiplication in the context of electronic structure applications for quantum mechanical simulation of molecules and materials from first principles. Accurate simulation of electronic structure, via the coupled-cluster [44] and many-body Green's function approaches, is feasible but expensive, i.e., such many-body methods have high-order polynomial operation and space complexity; for the foundational Coupled-Cluster Singles and Doubles method 
(CCSD), these are $N^{6}$ and $N^{4}$, respectively, with $N$ proportional to the system size. The high complexity limits the applicability of conventional (naive) formulations of predictive methods to systems with a few (5-10) atoms on a single workstation, and a few dozen (50-100) atoms on a supercomputer [35]. However, the recent emergence of robust fast/reduced-scaling formulations has greatly extended the applicability of such methods to hundreds of atoms on a single workstation in a matter of days [38]. Modern state-of-the-art HPC platforms should make it possible to deploy reduced-scaling coupledcluster (CC) methods with time-to-solution measured in minutes rather than in days.

The complex tensor algebra involved in the CCSD method can be reduced for our purposes to a single representative term, and usually the most expensive one (accounting routinely for $90 \%$ or more of the total work) ${ }^{1}$, often colloquially known the $A B C D$ term:

$$
R_{a b}^{i j}=\sum_{c d} T_{c d}^{i j} V_{a b}^{c d}+\ldots
$$

where the elements of tensor $T$ are the model parameters to be refined iteratively (in typically 10-20 iterations) to make tensor $R$ vanish. Tensor $V$ is fixed (does not change between iterations). Ranges of all indices are proportional to system size $N$, hence each tensor has $N^{4}$ space complexity, and the operation has $N^{6}$ operation complexity.

The tensor contraction in Equation (1) can be viewed as a multiplication of matrix $T$ (with fused indices $i j$ and $c d$ playing the role of row and column indices, respectively; in subsequent sections such matricized tensor $T$ will serve as matrix $A$ in $C=C+A B$ ) with square matrix $V$ (with $c d$ and $a b$ row and column indices; this will serve as matrix $B$ ). In practice the range of unoccupied indices ( $a b c d$ ) has rank $U$ that is a factor of 5-20 times larger than the corresponding rank $O$ of the occupied indices $i j$, hence transposes of matricized tensors $T$ and $R$ are tall-and-skinny matrices, with aspect ratios of 25-400.

In the conventional formulation of CCSD, all tensors are generally dense (modulo prefactor-reducing block-sparsity due to discrete geometric symmetries; here we only focus on block-sparsity due to dynamical structure of the physical problem that can lead to the reduction of complexity). The optimal formulation of dense matrix multiplication on distributed-memory systems [50], including for rectangular matrices [15], is relatively well understood and makes possible strongly scalable CCSD implementations [35, 46]. Extending these advances to reduced-scaling coupled-cluster variants in which tensors have complex block-sparse structure is nontrivial due to the physically-motivated nonuniform tiling of index ranges (e.g., it is not

\footnotetext{
${ }^{1}$ The permutational symmetries of tensors $T, V$ and $R$, which are essential for proper physics as well as attaining the optimal operation count, are neglected for simplicity.
} 
in general possible to partition the basis into even chunks without sacrificing locality) This leads to the loss of the near-perfect load balance that makes traditional communication-optimal algorithms attain strong scaling. Parallel computation with irregularly-tiled and/or data-sparse tensorial data structures is also a poor match to imperative, bulk-synchronous parallel programming style and execution models due to the irregular (and potentially dynamic) structure of the data. In this work, we demonstrate how these challenges can be addressed by modern task-based dataflow-style scheduling to achieve high performance on a distributed-memory heterogeneous cluster with multi-GPU nodes. The block-sparse evaluation of the ABCD term in Equation (1) in the so-called atomic orbital formulation will serve as the target performance benchmark; the reference CPU-only implementation of this term was developed in the open-source Massively Parallel Quantum Chemistry (MPQC) program [36].

\section{Design Principles}

We start with a detailed description of the problem in Section 3.1 before introducing the algorithm in Section 3.2. While the problem can be captured in terms of a rectangular matrix product, there are many characteristics that call for a new algorithmic approach.

\subsection{Problem Description}

As already mentioned, the problem is generated from a 4-dimension tensor, but can be viewed as a matrix multiplication, $C \leftarrow C+A B$, with the following characteristics:

1. The matrices are composed of heterogeneous tiles. This means that the size of the tiles strongly vary across rows and columns. On the positive side, the partitioning into tiles obeys the rules of matrix product: in the product of a tile row of $A$ and a tile column of $B$, the dimensions of the tiles are all compatible (as indicated in Figure 1). Unfortunately, many of these dimensions are too small to provide high computational intensity.

2. The matrices are block-sparse. This means that a significant fraction of the tiles in $A$ and $B$ are zero tiles (which opens the possibility for some tiles of $C$ to be zero tiles too). The non-zero tiles are dense, which is good news since efficient dense linear algebra GEMM kernels can be used for the non-zero tile products.

3. The matrices have very different sizes: $A$ and $C$ are short-and-wide, while $B$ is square. More precisely, $A$ has size $M \times K, B$ has size $K \times N$, and $C$ has size $M \times N$, where $M \ll K=N$ (typically $N=100 M$ ). 
As for tile indices, $A$ has $M^{(t)}$ tile rows (of various heights) and $B$ has $N^{(t)}$ tile columns (of various widths).

All these characteristics dramatically complicate the problem. As pointed out in Section 1, designing an efficient algorithm for matrix multiplication on multi-GPU accelerated distributed memory platforms is already a difficult task, even in the simple case where $A, B$ and $C$ are dense and square. For our problem, the heterogeneity of tile sizes further hardens the management of GPU memory and diminishes the peak performance of the kernels, while the sparsity decreases data reuse across different GEMMs.

The target platform is composed of $P$ processors, or nodes, each equipped with $g$ GPUs. We aim at executing the block-sparse matrix product on a $p \times q$ process grid, where $p q \leq P$. For square and dense matrices, the traditional algorithm uses a square 2D-grid with $p=q$, a 2D-cyclic distribution of the three matrices, and computes $C$ in place while $A$ and $B$ are communicated through the network. The significantly larger size of $B$ in front of that of $A$ and $C$ requires changing the traditional algorithm. In order to minimize the network traffic, we need to avoid circulating the largest of the matrices, so $B$ will be stationary. Technically, this amounts to simulating the product $B \leftarrow$ $A^{T} \times C$ and to perform a final reduction of $C$ tiles across grid columns. To avoid these costly reductions, an alternative is to distribute full columns of $B$ to processors, meaning that the distribution of $B$ becomes uni-dimensional on a flat $1 \times q$ grid (where $q=P$ ). Each column of $B$ is then entirely held by a single node, as opposed to partitioned across grid rows. However, this alternative is known to increase the communication volume related to $A$; this is why 2D-grids are generally preferred for matrix multiplication.

Yet another alternative is to duplicate the columns of $B$ and to use a $p \times q$ processor grid with $p \geq 2$. In this last solution, each grid row computes the product of an horizontal slice of $A$ by the whole matrix $B$. More precisely, $A$ is segmented into $p$ horizontal slices, and all $p$ grid rows work independently on their own slice, without any communication and in full parallelism. The price to pay is to replicate each column of $B p$ times in memory, one time per grid row, which puts pressure on CPU memory, but not on GPU memory which is the actual bottleneck for the computational perspective. We investigate this last solution and keep the number $p$ of grid rows as a trade-off parameter: using $p=1$ avoids the replication of $B$ but increases the communication volume of $A$; using $p \geq 2$ requires $p$ copies of each column of $B$ but decreases the communication volume of $A$ by a factor $p$.

\subsection{Algorithm}

The algorithm targets a 2D-grid of $p \times q$ processors, where $p$ is a parameter and $q=\left\lfloor\frac{P}{p}\right\rfloor$, where $P$ is the total number of available processors, so that 
$p q \leq P$. The matrix $A$ is distributed with a standard 2D-cyclic distribution. Let $A^{(k)}$ be the slice of $A$ distributed on row grid number $k$ where $0 \leq k \leq$ $p-1: A^{(k)}$ is composed of tile rows of $A$ of index $i$ such that $i \bmod p=k$. Let $C^{(k)}$ be the corresponding slice of $C$ (same row indices as $\left.A^{(k)}\right)$. Row grid number $k$ computes the product $C^{(k)} \leftarrow C^{(k)}+A^{(k)} B$. All these products are independent and are executed in parallel. Therefore, we focus on the description of the algorithm on a single grid row, and keep using $A$ instead of $A^{(k)}$ to ease notations. Recall that $A$ now has $\frac{M^{(t)}}{p}$ tile rows (assume $p$ divides $M^{(t)}$ for simplicity). To ease reading, we will denote the algorithm in terms of rows and columns, but remember that all operations are tiled, and we use row to denote a tile row and column to denote a tile column.

The main operation of the algorithm on a processor row of size $1 \times q$ is the following:

- Assign columns of $B$ to the $q$ processors, and on each processor partition assigned columns into blocks, using the load-balancing algorithm detailed in Section 3.2.1.

- On each processor in parallel, compute the column blocks one after the other. The size of a column block is monitored so that its size does not exceed $50 \%$ of a GPU memory. Hence each block will be transferred from the CPU to the GPU only once. See Section 3.2.2 for details.

- The operation within each block is segmented to avoid GPU memory overflow. Communications from CPU to GPU are carefully monitored throughout execution to limit the number of $A$ tiles transferred to GPU, in order to ensure that no tile of $B$ and $C$ is ever flushed back to CPU before all computations involving it, are completed. See Section 3.2.3 for details.

The cost of the algorithm is analyzed in Section 3.2.4.

\subsubsection{Column Assignment}

To load-balance the product $C \leftarrow C+A B$, let $f_{k}$ be the total number of floating point operations (flop) corresponding to column $k$ of $B$ in the product, for $1 \leq k \leq N^{(t)}$. We sort the columns by non-decreasing values of $f_{k}$ and assign them to the $q$ processors in a mirrored cyclic distribution: the first $q$ columns are assigned to the $q$ processors in that order, and the next $q$ columns are assigned to the $q$ processors in reverse order, and the process repeats every $2 q$ columns. The mirroring (reverse) pass is used to compensate the imbalance due to the initial forward pass.

Let $\mathcal{B}_{q}$ denote the subset of columns assigned to processor $q$. This processor will be in charge to compute the same columns of the product $C$. Note that $C$ will therefore follow the same row distribution as $A$ and the 


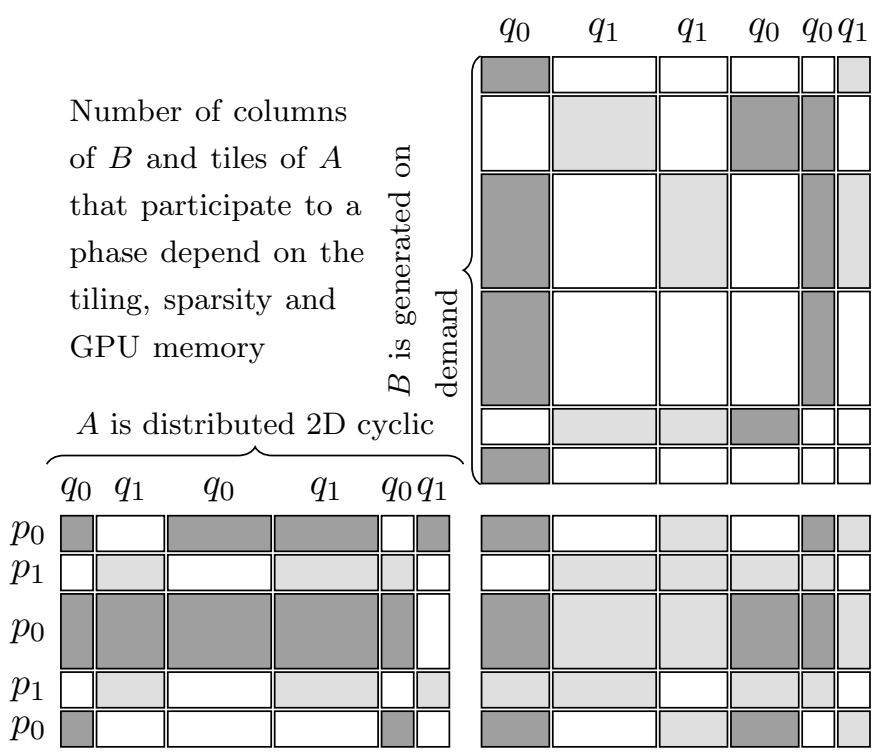

Figure 1: Representation of a phase of the algorithm for the process at the position $(p=0, q=0)$ in the process grid. Dark grey represent data loaded and used for computations by this process, light grey by other processes.

same column distribution as $B$. The assignment algorithm ensures that each processor receives a set of columns involving approximately the same amount of floating point operations, at the granularity of the columns of $B$, aiming at providing a good load-balance of the computations.

\subsubsection{Partition into Blocks}

Once the columns of $B$ have been assigned to the processors, they are divided into blocks which are assigned to GPUs. While the assignment of columns across nodes was intended to load-balance computations, the partitioning into blocks on each node aims at monitoring GPU memory usage. Locally, each processor computes a partition of its columns into blocks whose size fits in half the memory of one GPU. The goal is to enforce that each column of $B$, together with the local $C$ tiles in that column, will be transferred only once to the GPU. The algorithm sorts local columns ( $B$ columns assigned to the node) by non-increasing memory size (volume of data for the column and local $C$ tiles) and allocates these columns in that order to the GPUs, using a worst-fit algorithm. Each GPU starts with an empty block which is filled as the worst-fit algorithm progresses. A new block is created and assigned to a GPU in a round-robin fashion when the current column does not fit anywhere, in order to ensure no GPU is assigned more than one block than any other GPU.

During execution, blocks are transferred from CPU to GPUs in a blocking way: the transfer of the next block cannot start before operations on 
the current block are completed. This is to avoid new $B$ tiles flushing out current $B$ tiles still in use, which is critical for performance as experiences with regular square and dense problems show [22]. Again, the size of a block (including $C$ tiles) is computed so as not to exceed $50 \%$ of the GPU memory.

\subsubsection{Segmentation into Chunks}

There remains approximately $50 \%$ of GPU memory for $A$ tiles, depending on the space occupied by $B$ and $C$. How to organize the transfer of $A$ tiles to maximize re-use within a block? Say there are $c$ columns of $B$ in the block. We would like to work with groups of several rows of $A$ in parallel, say $r$ rows, and to segment the transfer of these tiles by chunks of $k$ tiles per row: this mimics the traditional algorithm that maximizes re-use by allowing $b$ chains of GEMMs to progress in parallel (one per column) and enforcing a total of $b r k$ GEMMS with only $r k$ transfers of $A$ tiles. The value of the chunk depth $k$ is computed for each new chunk of $A$ so that $r k$ tiles of $A$ fit in the remaining memory of the GPU. Unfortunately, there is no guarantee that such a nice re-use will be achieved for our problem, because of the sparsity pattern of the tiles. It may well be the case that a tile of $A$ is used only once instead of $c$ times in the block, if $c-1$ out of the $c$ potential products involving it are with zero tiles of $B$. Still, this is the best that can be done for re-use, and we implement this segmentation into chunks of $r$ rows of $A$. However, due to the heterogeneity of the tiles, we cannot load $k$ tiles per row any longer; instead, we build chunks greedily by adding one tile per row of $A$ in a cyclic fashion until half the remaining GPU memory, i.e., $25 \%$ of total GPU memory, is exhausted. The other half of remaining memory, i.e., the last quarter of total GPU memory, is saved to prefetch the next chunk of $A$ tiles, to increase the overlap of communications with computations. Owing to this careful GPU memory management, chunks can proceed with minimal gap due to communications of $A$ tiles, and without any flushing of $B$ and $C$ tiles back to CPU memory.

\subsubsection{Analysis}

The inspection phase required by the algorithm before execution has a cost $O\left(\left(N^{(t)} \log N^{(t)}+n n z_{B}\right)\right)$, where $n n z_{B}$ is the number of non-zero tiles in matrix $B$. This is linear in $n n z_{B}$, which is the dominant number of tiles in the matrix product. Indeed, for column assignment, there are $N^{(t)}$ columns in $B$; computing the weight of each column is $O\left(n n z_{B}\right)$, and sorting the columns by weight is $O\left(N^{(t)} \log N^{(t)}\right)$. For partitioning into blocks, the algorithm needs to compute the memory requirement of each column, which is also $O\left(n n z_{B}\right)$; then the allocation of columns into blocks is linear in $N^{(t)}$. Finally, chunking the blocks is linear in the number of non zero $A$ tiles. As the number of non-zero $B$ tiles is larger than the number of non-zero $A$ tiles, 
this can be ignored in the evaluation. Altogether, the overhead induced by the algorithm is of the same order as the number of non-zero $B$ tiles, and has a negligible cost on execution.

To minimize the amount of communication between nodes our algorithm assigns tile contraction tasks to the processor where the required tile of the largest matrix, $B$, is located; in other words, matrix $B$ is stationary and only the tiles of matrices $A$ and $C$ are communicated. There are thus only initial and final communications that deploy tiles of $A$ where they are needed, and then return the tiles of $C$ where they are required. The exact amount of communication is data-dependent: it depends not only on the sparsity of the communicated matrices $(A$ and $C$ ) but also on the sparsity of the stationary matrix $B$, since based on the latter a given tile of $A$ may not be needed on all nodes of its processor grid row. Also, some tiles of $C$ might not need to be moved, depending on the outcome of the load-balancing phase. To provide a bound on the amount of communication, consider the worst case of fully dense matrices: for a $p \times q$ processor grid, each tile of $A$ will then be needed on $q-1$ nodes as an initial communication, and each tile of $C$ produced might need to move to another node. In the worst case, the matrix $A$ needs to be broadcast to $q-1$ nodes, and the entire matrix $C$ needs to move. Note that there are no movements of $B$ between nodes, only between CPU memory and GPU memory, and that the communication, both initial for $A$ and final for $C$ happen in parallel with computation (as soon as a tile of $A$ is received, computations can occur, and as soon as a computation on $C$ is complete, it can be communicated back to its final location in CPU memory).

\section{Implementation}

This algorithm has been implemented using an inspector-executor strategy over the Parameterized Task Graph (PTG) language [13] over the PaRSEC runtime system [9]. The implementation is available at [23]. PaRSEC is a distributed task-based runtime system that targets large scale heterogenenous systems. Task programming has multiple advantages for exploiting large scale hybrid platforms to deploy complex algorithms: the runtime system that schedules the work has multiple choices of tasks to execute at any time, allowing it to adapt the execution to the circumstances; taskification of the work allows to isolate computational kernels that can be coded with a variety of alternative implementations targeting different hardware (e.g., CPU and GPU devices); task representation, coupled with a distributed runtime, also separates the computations from the communications in entities that can be managed by the runtime system: instead of embedding the communications within the control flow of the program, basically enforcing to adapt the program to the platform, the algorithm is expressed at a higher 
level, allowing more performance portability over different setups.

PTG is a domain-specific language that targets affine algorithmic descriptions, such as those used in dense linear algebra. The DPLASMA library is entirely written with it [16]. The idea behind PTG is to define the DAG Directed Acyclic Graph) of tasks as a concise and parameterized collection of tasks that exchange data through flows. Tasks are defined using task classes (a rudimentary templating approach), and task classes express synthetic conditions to enable input and output flows that carry the data. When the algorithm is regular, these conditions are fixed by a few parameters of the problem (e.g., the input matrix size, the tile size). In our case, however, the problem is irregular, both because the matrices are block-sparse and because they are irregularly tiled.

Thus, an inspector phase computes first what tasks exist, and how the data must flow between them. Then, a generic PTG that takes as input an execution plan produced by this inspector phase, allows the runtime system to execute it. This is sufficient to obtain a correct implementation of the irregular block-sparse matrix product. However, in order to implement the algorithm described above, one needs to be able to control the flow of data across node boundaries; so we introduce, in addition to the necessary data flow, a control flow. The control flow does not change the correctness of the algorithm, but increases its performance by preventing the scheduler of the runtime system to take wrong decisions (e.g., selecting a GEMM that is ready but that requires to eject some data that could be reused from that GPU memory), and forcing it to follow the strategy described in Section 3.2. Thus, the algorithm representation can be seen as the superposition of two DAGs, having the same nodes (the tasks) but different sets of edges. One DAG, the dataflow DAG, represents the tasks and the data flow between them, a pure dataflow description of the algorithm as an unhindered rendition of the potential parallelism. The second DAG, the control DAG, represents a set of performance constraints, that are architecture specific, and that are necessary for the runtime to provide a finer control of the existing parallelism, in order to constraint when data transfers happen. This is the way chosen to optimize the execution of the tasks represented by the dataflow DAG.

The control flow DAG is also expressed within the PTG, and depends on the GPU memory, and the sparsity of the input matrices. Thus, it is also computed during the inspection phase, and provided as part of the execution plan. Note, however, that communications between nodes and transfers between the main RAM to GPUs are not explicit: they are deduced from the dataflow and realized in the background (i.e., in parallel of task executions) by the runtime system. As a consequence, when the algorithm reserves $50 \%$ of a GPU memory to receive tiles of $B$ and $C$ when building a block, this is really implemented by constraining, with control flow, which tasks are ready to execute on that GPU, so they cannot refer more than $50 \%$ of the 
GPU memory if they were scheduled together on that GPU. Data transfers happen at the granularity of tiles, and tasks are scheduled as soon as the data they need is available on the GPU. The same applies to node-to-node transfers: although processes sharing the same row in the process grid need to have a copy of their share of the matrix $A$, this broadcast happens in the background, at the tile granularity, and tasks can be scheduled as soon as the data they need becomes available.

In addition to the data flow, PaRSEC programmers need to provide a description of the data to the runtime system. In our case, the matrices $A$ and $C$ are given using the data collections library available in PaRSEC. The matrix $B$, however, is stored implicitly: generation functions allow to instantiate any tile when needed. We extended PaRSEC's data collection library by developing a new data collection that instantiates the tasks corresponding to the tile generation on demand, when a tile needs to be instantiated. The usual mechanisms within the PaRSEC runtime system to manage the life-cycle of these data is then used to cache them as long as they are needed by any task, and discarded after this. The algorithm ensures that each tile of $B$ is instantiated at most once per node that needs it (as noted, columns of $B$ are replicated between processes that share the same column in the process grid), and since the generation routine does not have a CUDA implementation, these tasks are always executed on the CPUs.

Last, implicit data movement allows the runtime system to select the 'best' source of data, when multiple sources are available. This happens, for example, when two GPU devices need the same tile of $A$ in our algorithm. One GPU needs to pull it from main memory, paying the cost of a PCIExpress transfer. But the second GPU may use the copy residing on the first one, leveraging the fast NVlink to implement a device-to-device copy, thereby reducing the pressure on the PCI-Express bus to allow other memory transfers. This feature comes directly from the runtime system and does not require any modification of the algorithm itself.

\section{Performance Evaluation}

All performance measurements presented below were run on Summit, hosted at Oak Ridge National Laboratory. Summit holds 4,600 IBM AC922 compute nodes, each containing two POWER9 CPUs and 6 NVIDIA Volta V100 GPUs. The POWER9 CPUs have 22 cores running at $3.07 \mathrm{GHz}$, and 42 cores per node are made available to the application. Dual NVLink 2.0 connections between CPUs and GPUs provides a $25 \mathrm{~GB} / \mathrm{s}$ transfer rate in each direction on each NVLink, yielding an aggregate bidirectional bandwidth of $100 \mathrm{~GB} / \mathrm{s}$.

PaRSEC, the proposed GEMM implementation and the driver program were all compiled in optimized (Release) mode, using XLC 16.1.1-2, CUDA 
9.2.148, Spectrum MPI 10.3.0.0 available on the Summit programming environment. The BLAS3 GEMM kernel was the one provided in the cuBLAS library shipped with CUDA.

We measured the practical peak of the GEMM kernel in this version of cuBLAS and this hardware at 7.2Teraflop/s per GPU. To obtain this value, we ran a single GEMM operation on large matrices that were pre-initialized in the GPU memory, repeated the operation 10 times, and took the fastest run measured.

All performance evaluation results presented below are obtained by measuring the time of executing the implementation described in Section 4, with the matrix $A$ distributed between the nodes in a 2D-cyclic fashion, $C$ empty (the necessary tiles will be allocated and initialized to zero when needed), and $B$ generated on demand, on the cores. The time to generate $B$ and inspect the execution, as well as the time to move data of $C$ back and forth to the GPU are all taken into account in the measurements presented below. Thus, the cost of data movement from CPU to GPU memory is always included in our measurements. Moreover, it is important to notice that due to the targeted domain science, in most cases the matrices $A$ and $C$ are too large to fit in GPU memory.

Each point is measured 5 to 10 times, and all figures showing performance present a Tukey box plot at the mark. On most figures, the measured variability is so small that the box plot is hidden by the mark or the line placed at the mean value, highlighting the stability of the distributed algorithm.

\subsection{Synthetic Benchmarks}

First, we consider matrices with random sparsity, in order to understand the performance of the implementation in a controlled setup. We set the number of nodes to 16 , and start from a square and dense problem $(M=K=N)$, then increase $N$ and $K$ (keeping $K=N$ to mimic the aspect ratios of the matrices involved in the target coupled-cluster ABCD contraction), and also decrease the density. Irregularity of tiling is set randomly to be uniform between 512 and 2048 (in each dimension), and both input matrices ( $A$ and $B$ ) have the target density (the density of $C$ being computed from the shape and non-zero tiles of $A$ and $B$ ). To decide which tiles are zero in $A$ and $B$, an iterative algorithm selects uniformly a non-zero tile to eliminate, until eliminating another tile would draw the density of the matrix (element-wise) under the threshold.

Figure 2 depicts the performance as a function of $N, K$ and the density of the problem. Several conclusions can be drawn. First, the performance is highly dependent on the density of the problem; in fact, the density has more impact than the problem size or shape. This is expected as a lesser matrix density provides less opportunity for data reuse, shifting the blocksparse GEMM from compute-intensive to data-intensive (in this instance 


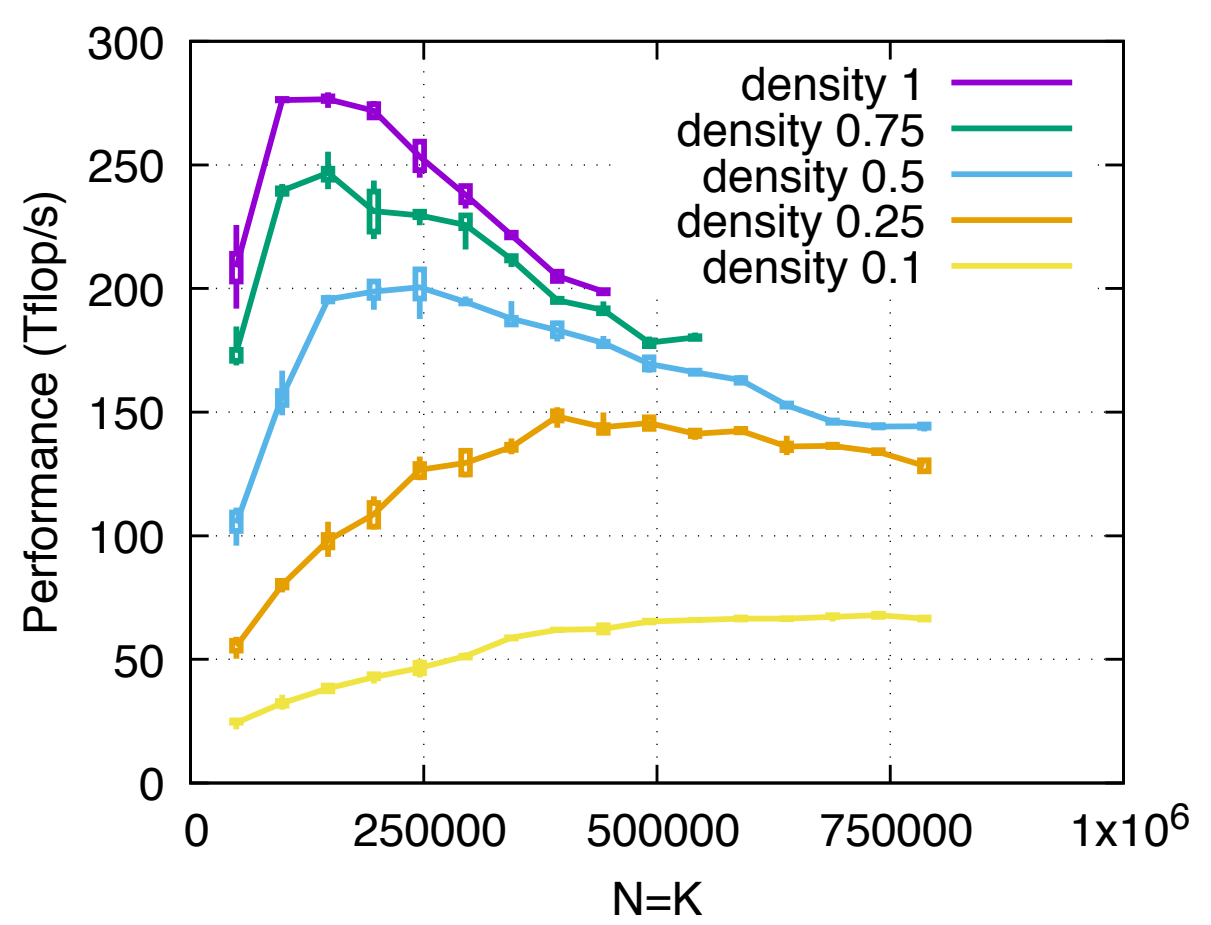

Figure 2: Performance as a function of the matrix size $(N$ and $K)$ and density, on 16 nodes of Summit. Peak performance of GEMM for the 16 nodes is estimated at $672 \mathrm{Tflop} / \mathrm{s}(16 \times 6 \mathrm{GPU} \times 7 \mathrm{Tflop} / \mathrm{s})$

GPU transfer-intensive). We will revisit this topic later.

Second, the performance even in the dense case reaches only half the GEMM-peak of the GPUs. Comparing with the results that were obtained in [22] on the same machine, using the same runtime system, at this problem size and number of nodes, $80 \%$ to $90 \%$ of the GEMM-peak should be achievable. This difference is due to the problem shape, which required a different algorithm: tiles of $B$ are generated on demand, but the size of $B$ does not allow (in the application case) to keep all of them in memory until the completion of the algorithm. It is thus necessary to minimize the number of times that tiles of $B$ are generated, and this drives the design of the algorithm to work on columns of $B$, while the traditional GEMM algorithms for square matrices, e.g., $[22,45]$ work on square submatrices of $C$. As a consequence, the algorithm is not designed to perform optimally on square dense problems. As the $A$ and $C$ matrices become short and wide, the algorithm becomes more efficient, but the shape of the matrices themselves reduce the amount of reuse for the tiles of $A$ and $C$, and thus limits the performance achievable in the dense case.

Last, as the algorithm requires most tiles of $A$ to be replicated on the processes that share the same row position in the process grid, these data 


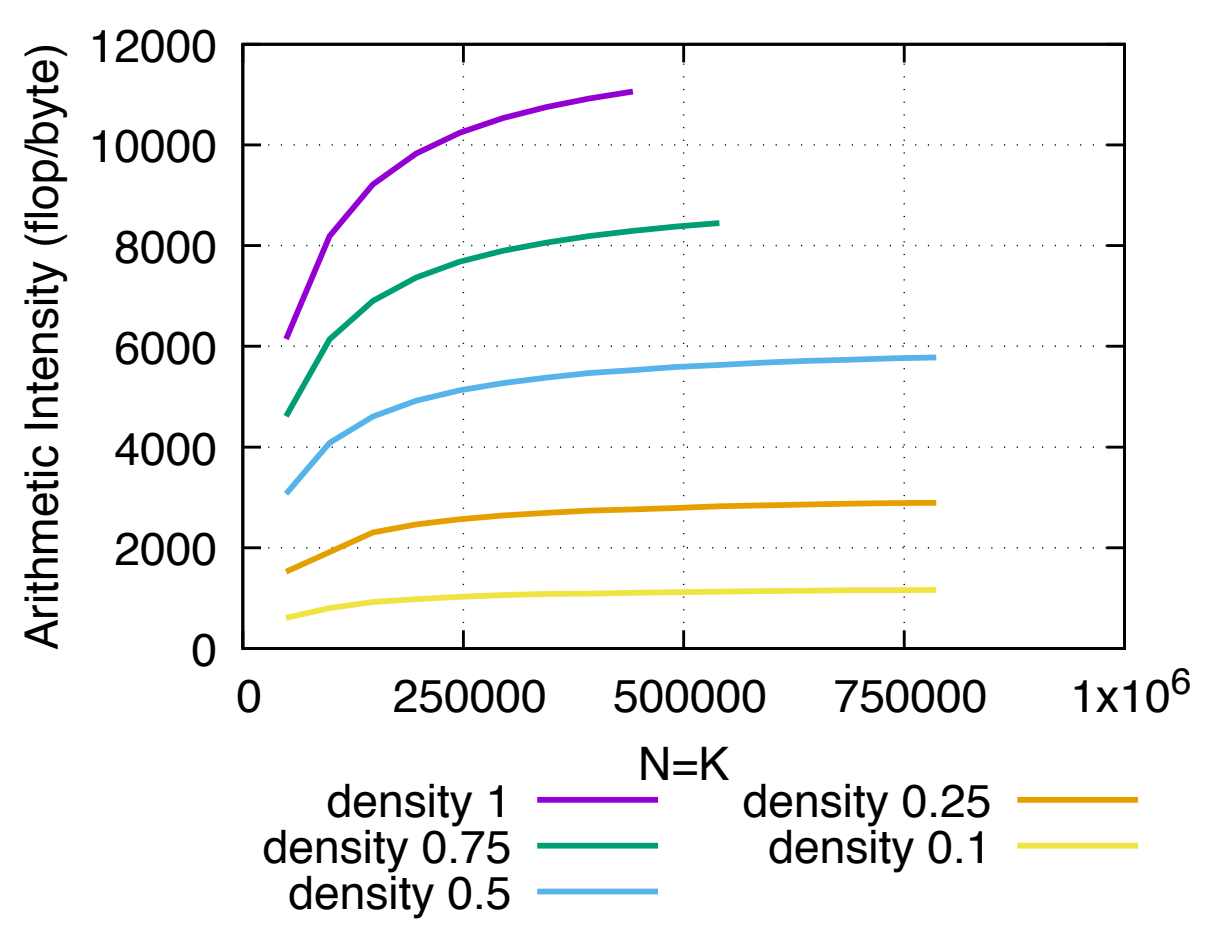

Figure 3: Theoretical arithmetic intensity of the synthetic matrix multiplication problem, as a function of size parameters $(N$ and $K)$ and density, on 16 nodes of Summit.

broadcasts are expensive relative to the number of floating point operations, when the problem is square. In that case, the processes start by computing products with the tiles of $A$ that are local, but if those are not enough to completely overlap the communications, execution stalls until the required tiles are received. When $N$ increases, the number of operations available to overlap this communication increases, allowing the algorithm to reach higher performance. This increase in operations / bytes is illustrated in the arithmetic intensity (Figure 3).

Increasing $K$ causes the tiles of $A$ to be transferred to the GPU multiple times, as the part of $A$ assigned to each GPU plus the column of $B$ do not fit on the GPU memory anymore. This reduces the performance by increasing communication costs and reducing the effective arithmetic intensity. The maximum arithmetic intensity (i.e., number of floating point operation divided by the aggregate size of $A, B$, and $C$ ) is depicted in Figure 3 . The maximum intensity is an upper bound on the effective intensity since it can only be realized if $A, B$, and $C$ were loaded only once to the device memory. As seen previously, the algorithm needs to load tiles of $A$ multiple times, as the available memory on the GPU does not allow to keep all the input data, effectively decreasing the arithmetic intensity. Figure 3 also provides 


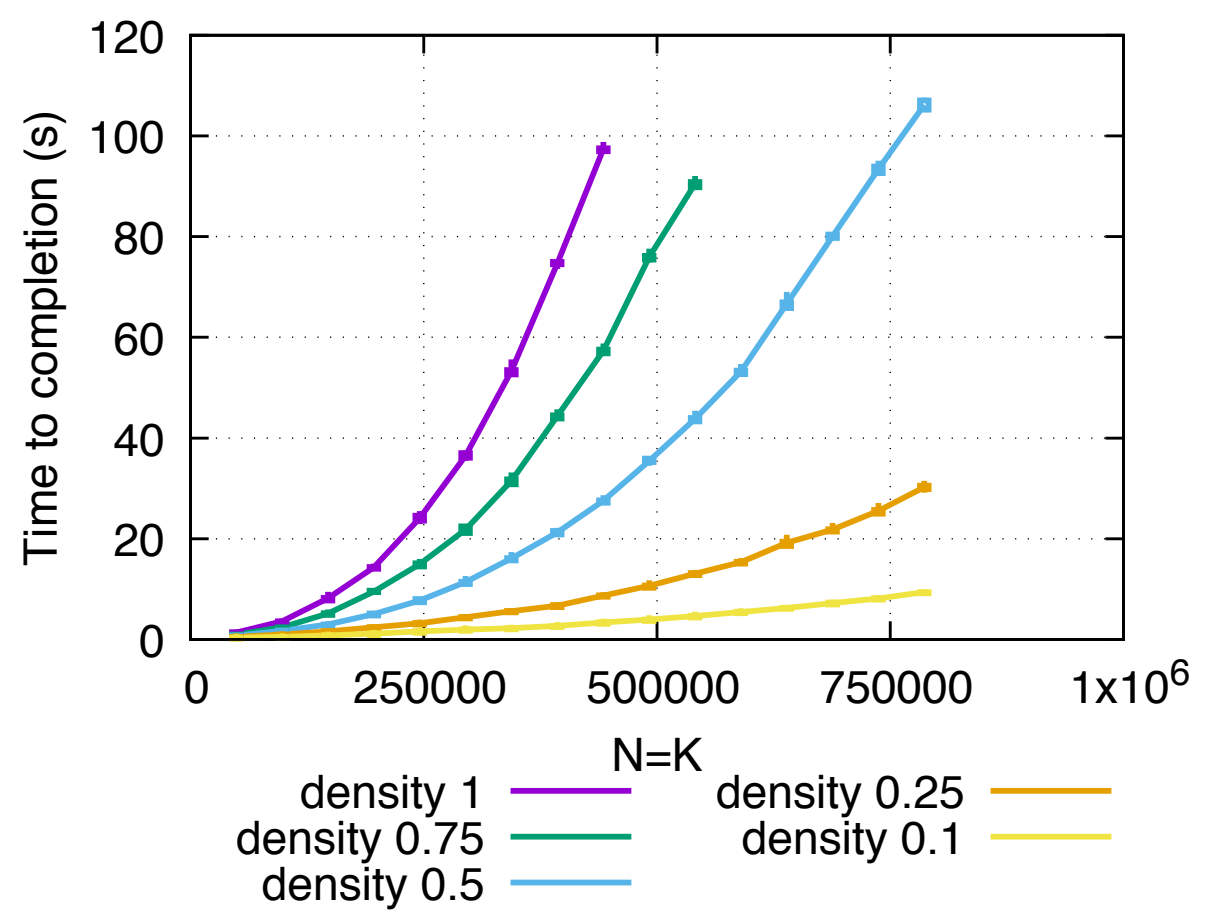

Figure 4: Time to completion of the synthetic problem, as a function of matrix size $(N$ and $K)$ and density, on 16 nodes of Summit.

an explanation for the performance increase at the beginning of the curves in Figure 2, when columns of $B$ and rows of $A$ can fit together on the GPU, and also explains why the dominating element for the performance is the density of the matrices: as the sparsity increases, the number of operations relative to the amount of data to load decreases significantly, and as could be expected, the problem shifts from a compute-intensive problem to a dataintensive problem. In addition to this, each tile loaded on the GPU has a lower chance to get re-used for another product, as the number of tiles in the other matrix that correspond to it decreases with the density.

Although the effective arithmetic intensity and the measured performance inevitably decrease with the density of the problem, the time to solution remains dominated by the number of operations; since the latter decreases faster than the performance, as is illustrated in Figure 4, the time to solution also decreases with the density.

\subsection{Practical Example: Evaluation of the ABCD coupled- cluster tensor contraction for molecule $\mathrm{C}_{65} \mathrm{H}_{132}$}

In this section, we use the new implementation of block-sparse matrix multiplication to evaluate the time-determining step of the CCSD electronic 
structure model (Equation (1)). Since problem sizes and traits vary greatly in practical applications, we decided to use an example that would be most challenging for reaching high absolute performance, namely a quasi1-dimensional system and small atomic orbital (AO) basis, where the sparsity of tensors is maximized while the optimal (from the data compression perspective) tile size is small. The molecule we chose, $\mathrm{C}_{65} \mathrm{H}_{132}$, is representative of applications to 1-d polymers and quasi-linear molecules (such as some proteins); the choice of the def2-SVP AO basis is representative of medium-precision simulations in chemistry and condensed phase.

The ABCD term was evaluated using the AO-based formalism [26]. The input tensor $T$ representing its initial state in the coupled-cluster simulation was evaluated in AO basis using the Laplace transform approximation, with the occupied orbitals localized and both occupied and AO basis clustered to group spatially-close orbitals together [29]; the clustering defines tiling of the corresponding index ranges. The CPU-only implementation in MPQC evaluates tensor $V$ on the fly, as needed; due to the lack of publicly-available efficient kernels for direct evaluation of AO integrals on GPUs (such kernels are under development by some of us) the GPU benchmarks used blocksparse $V$ with the actual sparsity pattern determined by the CPU-only code but the tiles filled with random data. The sparse "shape" of tensor $R$ was determined from the sparse shapes of tensors $T$ and $V$ as described previously [10].

Due to the quasi-1-dimensional structure and compact basis the $T$ and $V$ tensors in Eq. (1) are indeed very sparse (Figure 5). Note that the index range extents $O=196$ and $U=1570$ are much larger than would be practical for conventional CCSD: using dense tensors, the operation count for the ABCD term evaluation would be $2 O^{2} U^{4} \approx 0.47$ Exaflop, whereas the use of sparsity allows to evaluate this contraction in $\approx 1$ Petaflop (see Table 1 ). Reduction of the operation cost by more than two orders of magnitude illustrates the power of reduced scaling formulations of the electronic structure methods; clearly, the only way to deploy efficiently accurate electronic structure methods on leadership-scale machines is to focus on the reduced-scaling formalisms.

Unlike element-sparse representation, block-sparse representation of tensors introduces an additional degree of freedom, namely tiling of the index ranges. The tiling has dual purpose, to maximize the degree of sparsity and to control performance traits such as the amount of data parallelism for tile-level kernels and the amount of task-level parallelism for tensorlevel operations. Namely, using tiles that are "too" large will reduce the degree of sparsity (in the limiting case of 1 tile per dimension the representation is dense) and greatly increase the operation count; using tiles that are "too" small will decrease the amount of data parallelism exploitable by the tile-level kernels (in the limiting case of 1 element per tile, the representation becomes element-sparse, typically used for sparse matrix computation) 


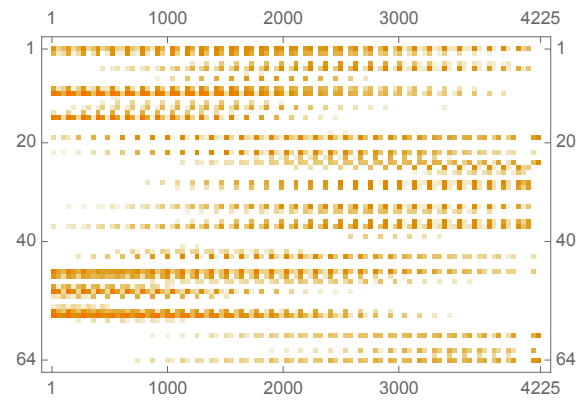

(a) $T$

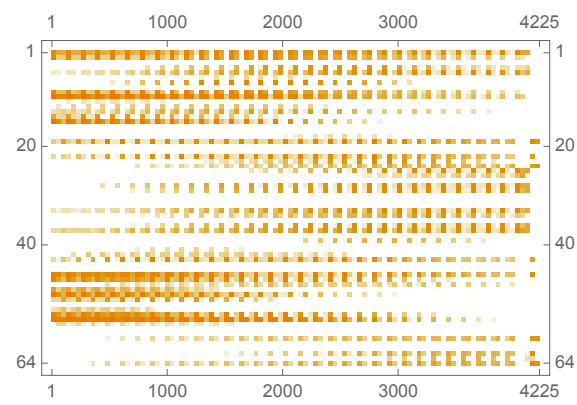

(c) $R$

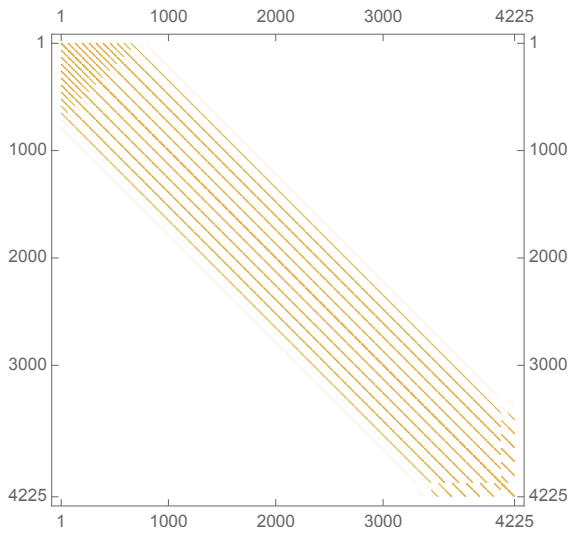

(a) $V$

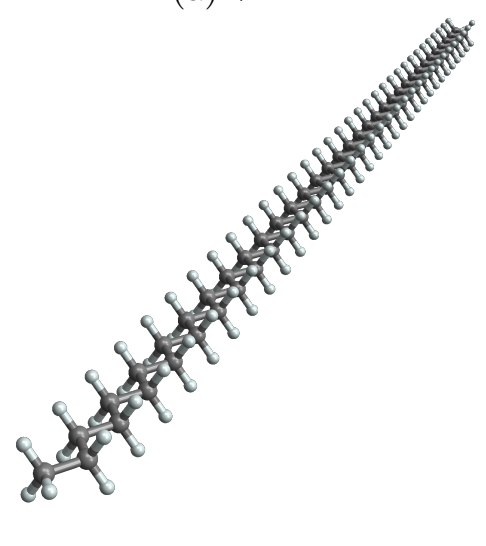

(d) the $\mathrm{C}_{65} \mathrm{H}_{132}$ molecule

Figure 5: Pictorial representation of matricized block-sparse tensors $T, V$, and $R$ for the $\mathrm{C}_{65} \mathrm{H}_{132}$ example (tiling $v_{1}$ is shown, with the aspect ratio is adjusted to make each tiles appear square). The extreme sparsity of the tensors is due to the quasi-one-dimensional shape of the molecule.. 


\begin{tabular}{l|l|l|l|l} 
& Tiling $v_{1}$ & Tiling $v_{2}$ & Tiling $v_{3}$ \\
\hline$M \times N \times K$ & \multicolumn{2}{|c}{$26576 \times 2464900 \times 2464900$} \\
\hline$\#$ flop & 877 Teraflop & 923 Teraflop & 1237 Teraflop \\
\hline \#flop (opt.) & 850 Teraflop & 899 Teraflop & 1209 Teraflop \\
\hline \#GEMM tasks & 1899971 & 468368 & 67818 \\
\hline \#GEMM tasks (opt.) & 1843309 & 455159 & 66315 \\
\hline Average \#rows/block & 700 & {$[500 ; 2500]$} & {$[1000 ; 5000]$} \\
\hline Average \#columns/block & 700 & {$[500 ; 2500]$} & {$[1000 ; 5000]$} \\
\hline Density of $T$ & $9.8 \%$ & $10.2 \%$ & $13.2 \%$ \\
\hline Density of $V$ & $2.4 \%$ & $2.6 \%$ & $3.1 \%$ \\
\hline Density of $R$ (opt.) & $14.9 \%$ & $16.1 \%$ & $21.7 \%$
\end{tabular}

Table 1: Relevant problem traits for the $\mathrm{C}_{65} \mathrm{H}_{132}$ test case with the three variants of tiling.

These two objectives are mutually contradictory, thus in practice for models with user-controllable tiling like the AO-basis CCSD, the tiling should be optimized to balance its effects on the operation count and performance.

To evaluate the impact of the tiling on performance, we consider three representative tilings of the index ranges. Since the k-means-based clustering algorithm that determines the range tilings is quasirandom [29] and cannot ensure uniform tiling (this would necessarily violate locality in all practical applications), these tilings are generated by specifying the target number of clusters for each index range. Table 1 summarizes the difference between the three different tilings, from the most fine-grained one $\left(v_{1}\right)$ to the most coarse-grained one $\left(v_{3}\right)$. Tiling granularity impacts the tile size and the sparsity of the problem: a large grain tiling provides more irregular but larger average and minimum tile sizes, and increases the number of computations, as illustrated in the table and in Figure 6 .

Figure 7 shows the execution time the ABCD contraction (Eq. (1)) for the $\mathrm{C}_{65} \mathrm{H}_{132}$ test case with the three tilings using between 3 and 108 V100 GPUs on Summit. Dotted lines represent a perfect strong scaling with respect to the 3 GPUs computation (first point). Time to solution decreases with the number of GPUs, from $272 \mathrm{~s}$ at 3 GPUs for $v_{1}$, down to $34.9 \mathrm{~s}$, at 108 GPUs. Similar trends are observed for the other tilings. The parallel efficiency is not 1 , however, as can be observed by the difference with the theoretical scaling curve: for $v_{1}$, at 108 GPUs, that parallel efficiency is down to $21 \%$, when it is higher for $v_{2}(36.5 \%)$, or $v_{3}(35.2 \%)$. The cost of broadcasting tensor $T$, which is needed on all ranks that share the same rows, grows with the number of nodes and thus limits the scalability of the approach due to the compute time on each node becoming comparable to the communication time.

More interestingly, we observe that the overall time to completion in the cases $v_{2}$ and $v_{3}$ are similar, while for tiling $v_{3}$ the contraction involves $34 \%$ more flops compared to tiling $v_{2}$ ! Both tiling choices lead to significantly lower time to solution than the most fine-grained tiling $v_{1}$, which has the 

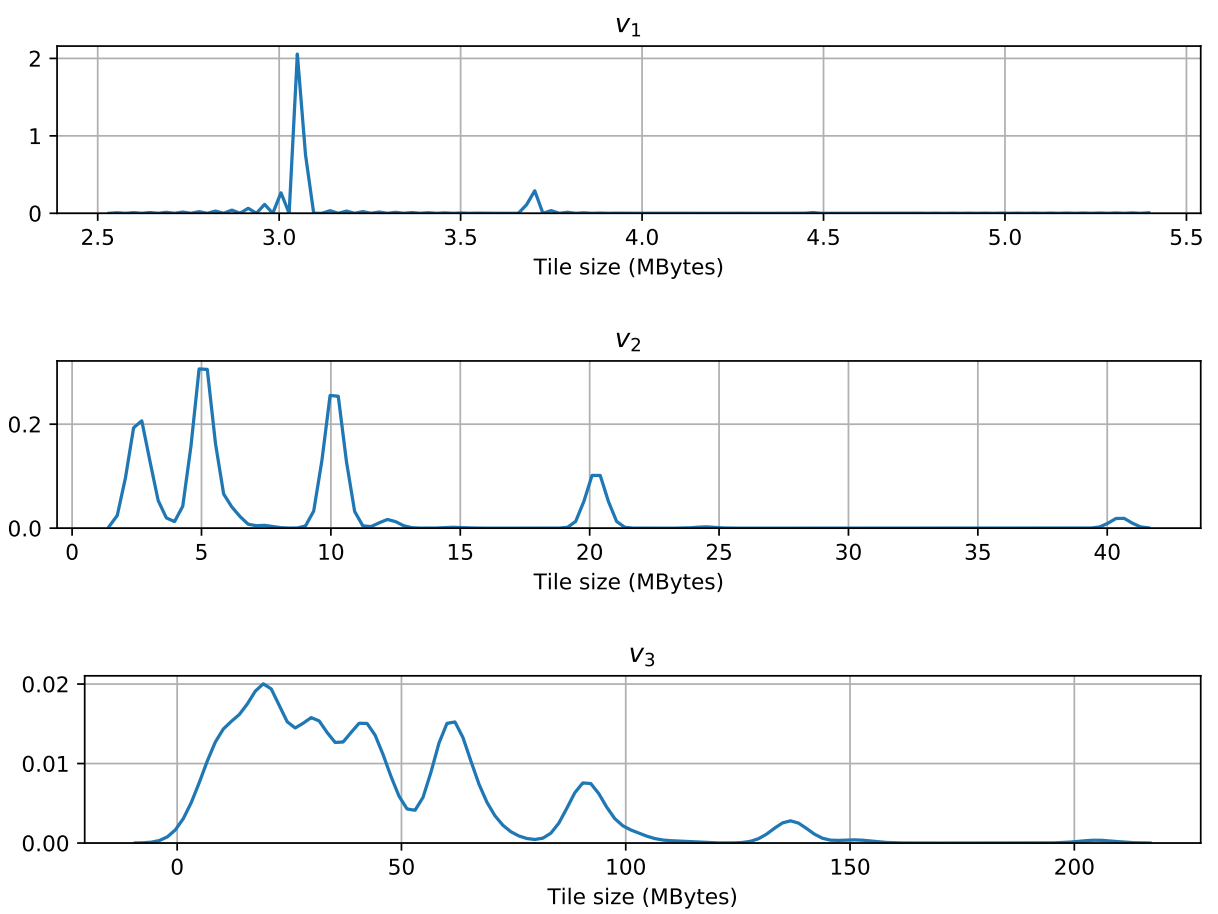

Figure 6: Tile size distribution for the three tilings of the $\mathrm{C}_{65} \mathrm{H}_{132}$ test case. All input matrices use a similar block distribution..

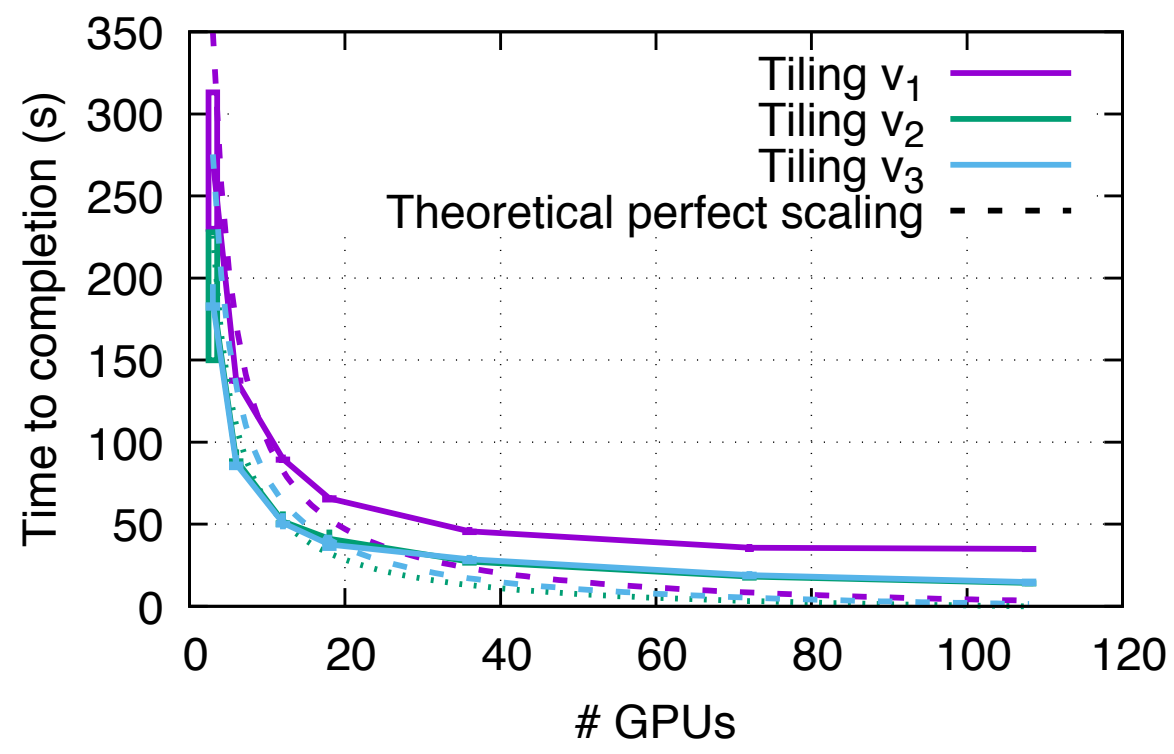

Figure 7: Time to completion for the $\mathrm{C}_{65} \mathrm{H}_{132}$ test case.. 


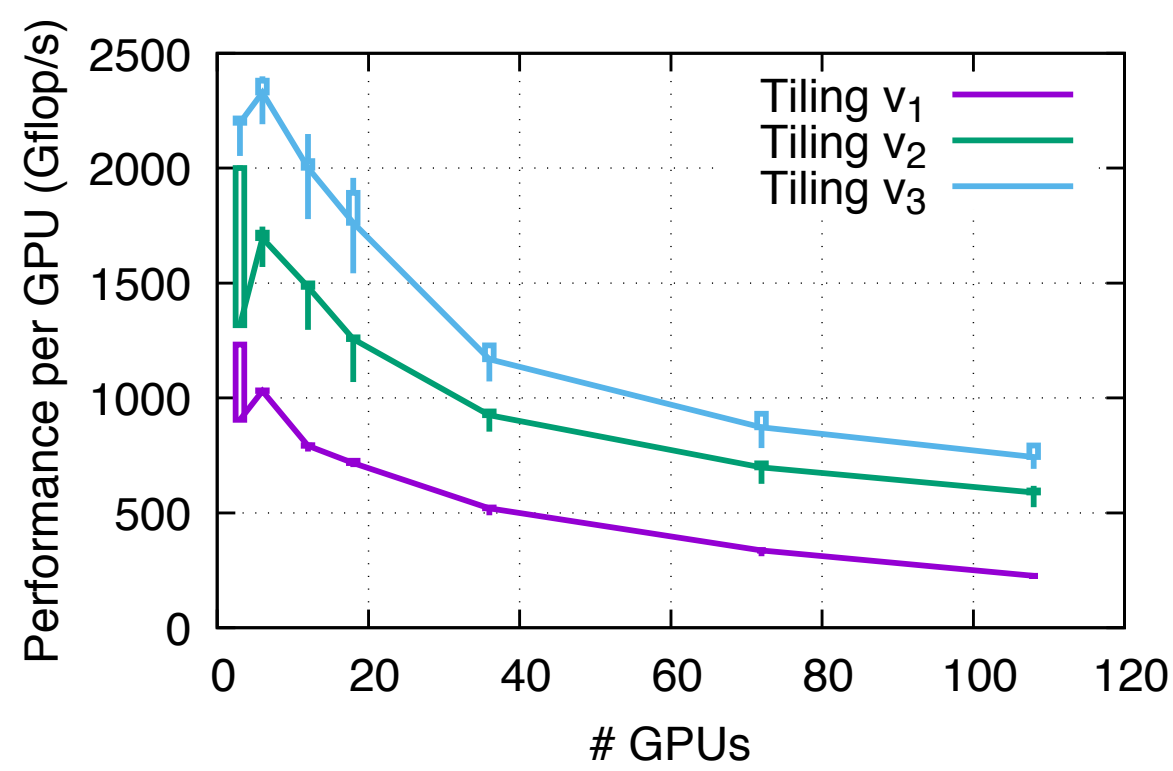

Figure 8: Performance per $G P U$ for the $\mathrm{C}_{65} \mathrm{H}_{132}$ test case.

lowest flop count of all three tilings. This is a good demonstration of the dual aspect of tiling: larger tiles lead to higher performance of tile-level kernels but reduce the amount of sparsity and thus increase the operation count. This is justified by Figure 8, which shows the performance per GPU in the same experiment. Clearly, by increasing tiling it is possible to trade sparsity for performance; the problem of how to determine the optimal tiling is left to future studies.

The performance per GPU follows an inverse trend with the tiling size: as tiles grow bigger $\left(v_{3}\right)$, each GPU kernel involves more flops. Moreover, the practical peak performance of these GPUs is around 7 Teraflop/s, while we observe up to 2.5 Teraflop/s for the $v_{3}$ tiling, which represents $35 \%$ of the peak performance, degrading to $11 \%$ at 108 GPUs (a 30\% parallel efficiency, as noted before). This shows that the arithmetic intensity (number of computation per bytes loaded) is too low to fully exploit the GPUs. Since a peak performance on a single tile can be obtained for tiles of $728 \times 728$, which is around the average tile size for tiling $v_{1}$, the problem does not reside in the tile sizes themselves, but in the tile re-use: the sparsity of the matrices $V$ and $T$ keep the re-use of data loaded on the GPU to a low amount, and GPU I/O dominates the execution time. Similar trends are observed in [43], where the performance at scale goes down to 30 Gflop/s per node (representing 3\% of the GEMM-peak of the machines used in this work).

As observed previously, the performance per GPU decreases with the number of GPUs, due to the added number of nodes involved that introduce communications: when going from 3 to 6 GPUs, the computation can remain 


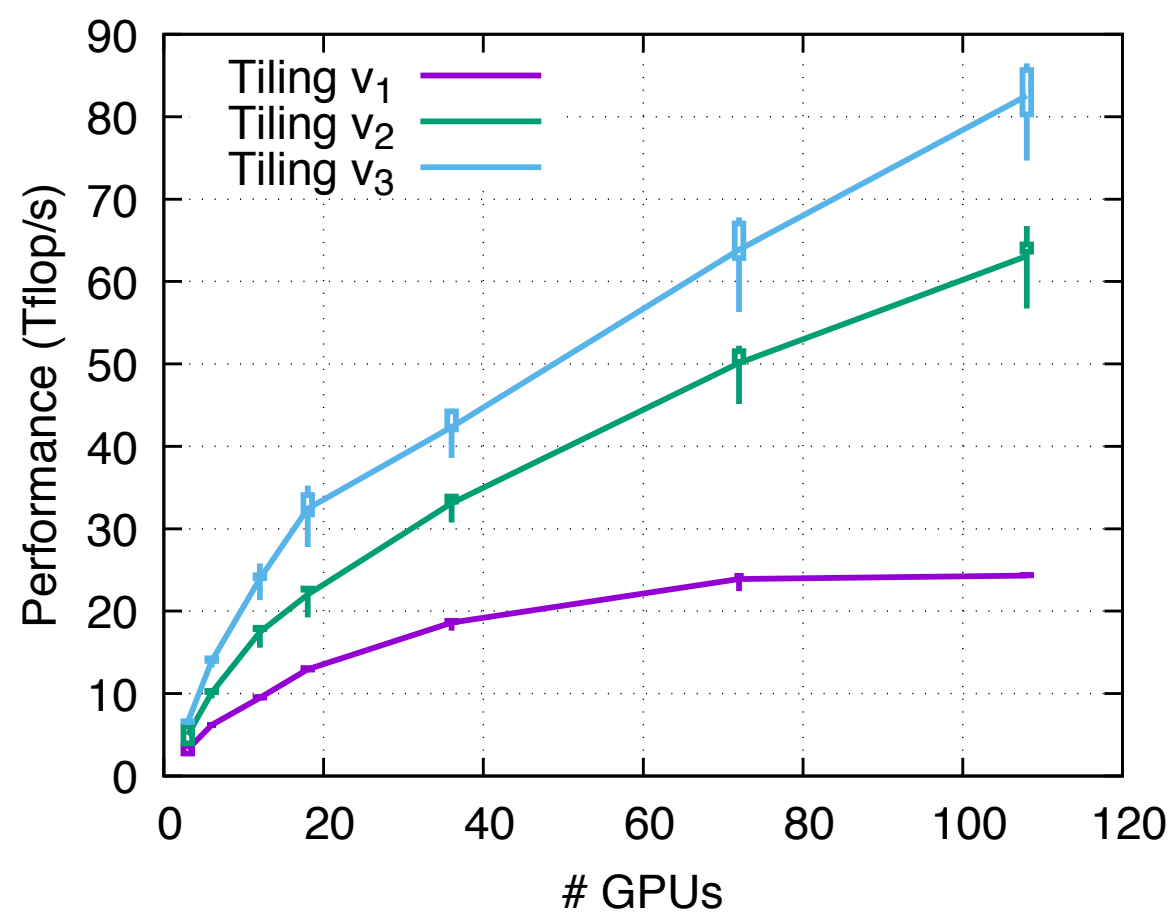

Figure 9: Performance for the $\mathrm{C}_{65} \mathrm{H}_{132}$ test case.

on the same node, and performance improves slightly; however upon further increase of the number of compute units, more nodes need to be introduced, increasing the total amount of communications. Figure 9, however, shows that overall, the performance continue to increase up to 108 GPUs, when the completion time is less than a minute, even for the finest grain case. Because GPU I/O dominates the performance per GPU, increasing the amount of computation (even significantly, when comparing $v_{2}$ and $v_{3}$ ) does not impact the time to solution, as these added computations can be done in parallel with the data transfers. In the worst case, reducing the computations, but also reducing data reuse by increasing sparsity $\left(v_{1}\right)$ increases the time to completion instead of decreasing it.

To compare the performance of the new GPU implementation of the state-of-the-art tensor contraction, we used the CPU-only code implemented in the MPQC package to evaluate the ABCD contraction for the $\mathrm{C}_{65} \mathrm{H}_{132}$ test case (no GPU version exists unfortunately). The computations utilizing $\{8,16\}$ nodes of Summit (total of 672 compute cores) completed in $\{308,158\}$ seconds, respectively. The corresponding GPU implementation using the most performant tiling $v_{3}$ on all GPUs available on the same set of nodes of Summit would reduce the time to solution by a factor $\approx 10$. The estimated efficiency of the CPU-only computation is rather low: assuming 2 Teraflop/s CPU peak performance per node for the 16-node job leads to 
an efficiency of $\approx 17 \%$ relatively to peak. Since the known performance heuristics of the CPU-only code in the MPQC package are established primarily for x86 architecture, it is likely that the CPU-only performance on Summit can be improved. Nevertheless the comparison is fair: MPQC is well-documented as a state-of-the-art coupled-cluster code $[34,35]$, and its CPU-only performance on Summit is an accurate reflection of the current state-of-the-art of chemistry codes on Summit.

\section{Related work}

\subsection{Matrix product on GPU}

The design of matrix product algorithms for high-performance computing platforms has received considerable attention in the recent years. Many libraries provide an implementation of matrix-product for distributed-memory machines $[16,18,19,33,41]$. Only a few libraries are capable of dealing with multi-GPU accelerated nodes: [11, 19, 22, 39, 45], and they currently suffer from the limitation that the stationary matrix (typically, chosen to be the result matrix $C$ ) must fit into the aggregate memory of the accelerators.

On the theoretical side, several authors have aimed at minimizing the number of communications for rectangular matrices of arbitrary sizes, since the seminal paper of Hong and Kung on the I/O pebble game [24]. Due to lack of space, we refer to a recent report [28] which provides a good overview and multiple references. Cache-oblivious algorithms are surveyed in [20,42]. Out-of-core algorithms for matrix product have been developed to optimize the number of transfers between hard disks and main memory. For dense square matrices, the pioneering work of Toledo [25,47] suggested to load three equal-size square blocks of $A, B$ and $C$ into main-memory, while a refined analysis [37] suggests to load the largest possible block of $C$, one slice of $B$ and to cycle tiles of $A$. The algorithm in [22] is an extension of this approach to multi-GPU accelerated platforms: a large block of $C$ tiles is loaded into GPU memory, allowing several chains of GEMMs to progress in parallel; but the chains are chunked to limit the number of $A$ and $B$ tiles that are loaded into GPU memory. Our algorithm for the block-sparse problem builds up upon these ideas, keeping the chunking and controlling GPU loads; but because of the huge size of $B$, the algorithm processes blocks of a few columns of $B$, and all corresponding $C$ tiles, as its main computational kernel.

\subsection{Block-Sparse Matrix Product on GPU}

There are few works directly targeting block-sparse matrix product on distributed systems using accelerators: [21] uses tensor flow [1] to implement a block-sparse matrix-product on a single GPU; SuiteSparse [14] includes 
matrix-product operations for block-sparse and sparse matrices, on single node GPU; $[39,40]$ present Chunk and Tasks, a distributed algorithm for block-sparse matrix product on GPUs, using quad-trees to represent the sparsity and reduce the memory overheads. This algorithm focuses on the product of square matrices, at scales that are much smaller than the problem considered in this paper. The key advantage of using quad-trees is to preserve data locality while reducing communications, and this for a wide range of sparsity patterns that arise in scientific applications. Our approach differs from theirs because we have to deal with matrices whose non-zero blocks occupy a space larger than the memory available on the GPUs. In our framework, the key to ensure that the tasks do not thrash the GPU bus is to introduce several additional levels of partitioning to constrain the execution order and to segment the update of each $C$ tile into chunks.

[43] introduced a CUDA-enabled version of Distributed Block Compressed Sparse Row (DBCSR) library, [7] a block-sparse matrix library used by the CP2K framework [12] (support for Xeon Phi accelerators also exists [6]). DBCSR originally targeted square block-sparse matrices, thus it uses the Cannon algorithm to schedule communications between nodes, reorders columns and rows to balance the work between nodes, and uses dynamic scheduling of work on the GPU to orchestrate computations. Matrices in CP2K typically have blocks of few particular sizes (this assumption does not apply to our data), thus DBCSR generates JIT-compiled optimized kernels for these particular block sizes. Ref [43] only demonstrated performance for square matrix multiplications of relatively modest size (the largest matrix size of $\sim 27 \mathrm{~GB}$ at $\sim 12 \%$ fill, compared to the largest matrix in our case of $\sim 1.5 \mathrm{~TB}$ at $\sim 3 \%$ fill). In the strong-scaling regime $\sim 30 \mathrm{Gflop} / \mathrm{s}$ per node $(<2.5 \%$ of the peak) were attained, although for smaller model problems $\sim 320$ Gflop/s can be attained $(\sim 27 \%$ of the peak, and roughly $3 \times$ faster than the non-tuned cuBLAS performance). DBCSR was recently generalized to tensor contractions, [45] which required introduction of modified versions of the Cannon algorithm with partial replication of data; however, the two target aspect ratios considered do not match ours, and no absolute performance data was presented.

To the best of our knowledge, our algorithm is the first algorithm published in the literature that is capable of minimizing the transfers from CPU to GPU memory for arbitrary matrix sizes and shapes, specifically targets multi-GPU nodes by taking advantage of the NVlink device-to-device communication capability when the opportunity arises, and leverages the shape of the large matrix to reduce node-to-node communications.

\subsection{Runtimes}

With the increase in hierarchy and complexity of the underlying hardware, maintaining a potential for high performance while abstracting the hard- 
ware to a simpler expression became critical. The literature is not short of proposals addressing this problem, including many evolutionary solutions that seek to extend the capabilities of current message passing paradigms with node-level features (MPI+X). A different, more revolutionary, solution explores task-based runtime systems as a substitute to both local and distributed data dependencies management. The ideas behind this are similar to the concepts put forward in workflows, parallelizing an algorithm by dividing it in sets of dependent tasks and organizing the data transfers to maximize the occupancy of most resources. But the scale, in terms of number and heterogeneity of resources, and the duration of the potential tasks set the new programming model well apart from workflows.

Thus far, dataflow runtimes have been used to either investigate irregular algorithms on shared memory (occasionally with accelerators), or, alternatively, to deploy dense, regular algorithms on distributed systems. This research provides a tangible base to address irregular applications that have so far been out of reach. To the best of our knowledge, no other runtime supports the expression of irregular tasks and communications with scalable implicit task discovery.

In this context, we are mostly interested in task-based runtime that simultaneously have the capability to run in a distributed-memory environment and support accelerators. Recent versions of the OpenMP specification [32] introduce the task and depend clauses which can be employed to express dataflow graphs, as well as explicit constructs target to offload computations on accelerators. Combined with an inter-node communication library, such as MPI or GasNet, it provide an interesting, but challenging programming ecosystem. StarPU [4] provides support for heterogeneous architectures, and covers distributed execution via the insertion of explicit communication tasks [2], which places the burden of organizing communication back on the application developer and on the communication library. Moreover, an efficient scheduling requires a training phase to highlight the classes of similar tasks, a luxury nonexistent in the target domain (tasks are variable in duration and data requirements). OmpSs [17], based on the Nanos++ runtime, follows a similar model to OpenMP, applications are annotated with directives to exploit the node-level parallelism supporting both CUDA and FPGA accelerators [8]. Legion [5], describes logical regions of data and uses those regions to express the dataflow and dependencies between tasks, and defers to its underlying runtime, REALM [49], the scheduling of tasks, and data movement across distributed heterogeneous nodes.

\subsection{Electronic Structure}

Distributed-memory algorithms for coupled-cluster and other many-body electronic structure methods have been in development since late 1980s 
and are now available in several packages (see [35] for a recent review of CCSD implementations), most notably in NWChem (a flagship distributedmemory quantum chemistry code), ACESIII, and GAMESS. Unfortunately very little of this capability can be executed on distributed-memory heterogeneous platforms. NWChem has a CUDA-based implementation of perturbative triples correction to CCSD, also known as (T), that has been demonstrated on a GPU-equipped distributed-memory platform and can take advantage of multiple GPUs and multiple CPU cores on each node (however, the CCSD code is CPU only) [30]. Very recently a distributed memory implementation of $(\mathrm{T})$ in $\mathrm{MPQC}$ was demonstrated that can take advantage of multiple GPUs per node [34]. GAMESS has demonstrated a GPU-capable implementation of select terms in the CCSD code on 1 node with 1 GPU [3].

\section{Conclusion}

In this paper, we focused on the block-sparse tensor contraction, a paradigmatic kernel in many scientific applications, whose characteristics (heterogeneity, sparsity, reduced computational intensity) make it a challenging candidate for distributed multi-GPU platforms. We have provided a highlytuned algorithm that carefully orchestrates task executions and data transfers between CPU and GPUs and between nodes to maximize resource occupancy. The flexibility and programmability of the underlying PaRSEC runtime greatly improved the algorithm implementation while providing a highly efficient support for multi-GPU distributed-memory platforms. The resulting implementation takes advantage of the GPUs, a major source of computing power, and obtains an efficiency and performance yet unrealized in the domain. Although comparison with existing tools to solve the same problem are not straightforward, because these tools do not run on the same hardware, the deployment on a real case shows a factor 10 of speedup using the same nodes. This shows that our new algorithm offers promising perspectives to solve problems of unprecedented scale and complexity.

Future work will aim at modeling the interactions between the tiling and the performance, in order to increase the efficiency of the algorithm. We will also extend the experiments to larger problems, representative of more complex molecular structures. Although we focused the evaluation on a representative of the most sparse cases, different molecules have the potential to provide much denser and compute-intensive input matrices, thereby (likely) enabling our algorithm to reach higher peak performance. 


\section{References}

[1] M. Abadi, P. Barham, J. Chen, Z. Chen, A. Davis, J. Dean, M. Devin, S. Ghemawat, G. Irving, M. Isard, et al. Tensorflow: A system for large-scale machine learning. In 12th $\{$ USENIX $\}$ Symposium on Operating Systems Design and Implementation (\{OSDI\} 16), pages 265-283, 2016.

[2] E. Agullo, O. Aumage, M. Faverge, N. Furmento, F. Pruvost, M. Sergent, and S. Thibault. Harnessing Supercomputers with a Sequential Task-based Runtime System, 2014.

[3] A. Asadchev and M. S. Gordon. Fast and Flexible Coupled Cluster Implementation. J. Chem. Theory Comput., 9(8):3385-3392, July 2013.

[4] C. Augonnet, S. Thibault, R. Namyst, and P. Wacrenier. StarPU: A unified platform for task scheduling on heterogeneous multicore architectures. Conc. Comp. Pract. Exper., 23:187-198, 2011.

[5] M. Bauer, S. Treichler, E. Slaughter, and A. Aiken. Legion: Expressing locality and independence with logical regions. In International Conference for High Performance Computing, Networking, Storage and Analysis, SC, 2012.

[6] I. Bethune, A. Gl”öss, J. Hutter, A. Lazzaro, H. Pabst, and F. Reid. Porting of the DBCSR library for sparse matrix-matrix multiplications to intel xeon phi systems. In S. Bassini, M. Danelutto, P. Dazzi, G. R. Joubert, and F. Peters, editors, Parallel Computing is Everywhere, volume 32 of Advances in Parallel Computing, pages 47 - 56. IOS Press, 2018 .

[7] U. Borštnik, J. VandeVondele, V. Weber, and J. Hutter. Sparse matrix multiplication: The distributed block-compressed sparse row library. Parallel Computing, 40(5-6):47-58, Apr. 2014.

[8] J. Bosch, A. Filgueras, M. Vidal, D. Jimenez-Gonzalez, C. Alvarez, and X. Martorell. Exploiting Parallelism on GPUs and FPGAs with OmpSs. In Proceedings of the 1st Workshop on AutotuniNg and ADaptivity AppRoaches for Energy Efficient HPC Systems, ANDARE '17, New York, NY, USA, 2017. Association for Computing Machinery.

[9] G. Bosilca, A. Bouteiller, A. Danalis, M. Faverge, T. Herault, and J. J. Dongarra. PaRSEC: Exploiting Heterogeneity to Enhance Scalability. IEEE Computing in Science Engineering, 15(6):36-45, 2013.

[10] J. A. Calvin, C. A. Lewis, and E. F. Valeev. Scalable task-based algorithm for multiplication of block-rank-sparse matrices. In IA3 '15, pages 1-8. ACM Press, 2015. 
[11] Chameleon. A dense linear algebra software for heterogeneous architectures. https://project.inria.fr/chameleon/, June 2020.

[12] CP2K. Open source molecular dynamics. https://www.cp2k.org, June 2020.

[13] A. Danalis, G. Bosilca, A. Bouteiller, T. Hérault, and J. J. Dongarra. PTG: an abstraction for unhindered parallelism. In Proceedings of the Fourth International Workshop on Domain-Specific Languages and High-Level Frameworks for High Performance Computing, WOLFHPC '14, New Orleans, Louisiana, USA, November 16-21, 2014, pages 2130. IEEE Computer Society, 2014.

[14] T. Davis et al. SuiteSparse : a suite of sparse matrix software. http: //faculty.cse.tamu.edu/davis/suitesparse.html, Apr 2020.

[15] J. Demmel, D. Eliahu, A. Fox, S. Kamil, B. Lipshitz, O. Schwartz, and O. Spillinger. Communication-Optimal Parallel Recursive Rectangular Matrix Multiplication. In 2013 IEEE International Symposium on Parallel \& Distributed Processing (IPDPS). IEEE, Jan. 2013.

[16] Distributed Parallel Linear Algebra Software for Multicore Architectures. DPLASMA. http://icl.utk.edu/dplasma.

[17] A. Duran, R. Ferrer, E. Ayguade, R. M. Badia, and J. Labarta. A Proposal to Extend the OpenMP Tasking Model with Dependent Tasks. Intl. Journal of Parallel Programming, 37(3):292-305, 2009.

[18] Elemental: $\mathrm{C}++$ library for distributed-memory linear algebra and optimization. Elemental. https://github.com/elemental/Elemental.

[19] M. Gates, J. Kurzak, A. Charara, A. YarKhan, and J. Dongarra. SLATE: Design of a Modern Distributed and Accelerated Linear Algebra Library. In SC'2019. ACM Press, 2019.

[20] K. Goto and R. A. v. d. Geijn. Anatomy of High-performance Matrix Multiplication. ACM Trans. Math. Software, 34(3):12:1-12:25, 2008.

[21] S. Gray, A. Radford, and D. P. Kingma. Gpu kernels for block-sparse weights. arXiv preprint arXiv:1711.09224, 3, 2017.

[22] T. Herault, Y. Robert, G. Bosilca, and J. J. Dongarra. Generic matrix multiplication for multi-GPU accelerated distributed-memory platforms over PaRSEC. In 10th IEEE/ACM Workshop on Latest Advances in Scalable Algorithms for Large-Scale Systems, ScalA@SC 2019, Denver, CO, USA, November 18, 2019, pages 33-41. IEEE, 2019. 
[23] T. Herault, Y. Robert, G. Bosilca, R. J. Harrison, C. A. Lewis, and E. F. Valeev. Distributed-memory multi-GPU block-sparse tensor contraction for electronic structure: software artifact. https://bitbucket. org/herault/irr-gemm-gpu-over-parsec, commit 17c88d2, April 2020 .

[24] J.-W. Hong and H. Kung. I/O complexity: the red-blue pebble game. In STOC '81: Proceedings of the 13th ACM symposium on Theory of Computing, pages 326-333. ACM Press, 1981.

[25] D. Ironya, S. Toledo, and A. Tiskin. Communication lower bounds for distributed-memory matrix multiplication. J. Parallel Distributed Computing, 64(9):1017-1026, 2004.

[26] R. Kobayashi and A. P. Rendell. A direct coupled cluster algorithm for massively parallel computers. Chem. Phys. Lett., 265(1-2):1-11, Jan. 1997.

[27] J. Kurzak, M. Gates, A. Charara, A. YarKhan, I. Yamazaki, and J. Dongarra. Linear systems solvers for distributed-memory machines with gpu accelerators. In Euro-Par 2019, pages 495-506, 2019.

[28] G. Kwasniewski, M. Kabić, M. Besta, J. VandeVondele, R. Solcà, and T. Hoefler. Red-blue pebbling revisited: near optimal parallel matrixmatrix multiplication. arXiv e-prints, page arXiv:1908.09606, Aug 2019 .

[29] C. A. Lewis, J. A. Calvin, and E. F. Valeev. Clustered Low-Rank Tensor Format: Introduction and Application to Fast Construction of Hartree-Fock Exchange. J. Chem. Theory Comput., 12(12):5868-5880, Dec. 2016.

[30] W. Ma, S. Krishnamoorthy, O. Villa, K. Kowalski, and G. Agrawal. Optimizing tensor contraction expressions for hybrid CPU-GPU execution. Clust. Comput, 16(1):131-155, 2013.

[31] Oak Ridge National Laboratory. Oak Ridge Leadership Computing Facility. https://www.olcf .ornl.gov/.

[32] OpenMP. OpenMP 4.0 Complete Specifications, 2013.

[33] Parallel Linear Algebra PACKage. PLAPACK. http://www.cs . utextas.edu/users/plapack.

[34] C. Peng, J. Calvin, and E. F. Valeev. Coupled-Cluster Singles, Doubles and Perturbative Triples with Density Fitting Approximation for Massively Parallel Heterogeneous Platforms. Int. J. Quant. Chem., 12(119):e25894, 2019. 
[35] C. Peng, J. A. Calvin, F. Pavošević, J. Zhang, and E. F. Valeev. Massively Parallel Implementation of Explicitly Correlated Coupled-Cluster Singles and Doubles Using TiledArray Framework. J. Phys. Chem. A, 120(51):10231-10244, Dec. 2016.

[36] C. Peng, C. Lewis, X. Wang, M. Clement, F. Pavosevic, J. Zhang, V. Rishi, N. Teke, K. Pierce, J. Calvin, J. Kenny, E. Seidl, C. Janssen, and E. Valeev. The Massively Parallel Quantum Chemistry Program (MPQC), Version 4.0.0. http://github.com/ValeevGroup/ mpqc, 2018.

[37] J.-F. Pineau, Y. Robert, F. Vivien, and J. Dongarra. Matrix product on heterogeneous master-worker platforms. In $A C M$ SIGPLAN PPoPP'2008, pages 53-62. ACM Press, 2008.

[38] C. Riplinger, P. Pinski, U. Becker, E. F. Valeev, and F. Neese. Sparse maps-A systematic infrastructure for reduced-scaling electronic structure methods. II. Linear scaling domain based pair natural orbital coupled cluster theory. J Chem Phys, 144(2), Jan. 2016.

[39] E. H. Rubensson and E. Rudberg. Locality-aware parallel block-sparse matrix-matrix multiplication using the chunks and tasks programming model. Parallel Computing, 57:87 - 106, 2016.

[40] E. H. Rubensson, E. Rudberg, and P. Sałek. A hierarchic sparse matrix data structure for large-scale hartree-fock/kohn-sham calculations. $J$. Computational Chemistry, 28(16):2531-2537, 2007.

[41] Scalable Linear Algebra PACKage. http://www.netlib.org/ scalapack.

[42] M. D. Schatz, R. A. van de Geijn, and J. Poulson. Parallel matrix multiplication: A systematic journey. SIAM J. Scientific Computing, 38(6):C748-C781, 2016.

[43] O. Schütt, P. Messmer, J. Hutter, and J. VandeVondele. GPUAccelerated Sparse Matrix-Matrix Multiplication for Linear Scaling Density Functional Theory, chapter 8, pages 173-190. John Wiley \& Sons, Ltd, 2016.

[44] I. Shavitt and R. Bartlett. Many-Body Methods in Chemistry and Physics: MBPT and Coupled-Cluster Theory. Cambridge Molecular Science. Cambridge University Press, 2009.

[45] I. Sivkov, P. Seewald, A. Lazzaro, and J. Hutter. DBCSR: A blocked sparse tensor algebra library. In Parallel Computing: Technology Trends, Proceedings of the International Conference on Parallel Computing, PARCO 2019, Prague, Czech Republic, September 10-13, 2019, 
volume 36 of Advances in Parallel Computing, pages 331-340. IOS Press, 2019.

[46] E. Solomonik, D. Matthews, J. R. Hammond, J. F. Stanton, and J. Demmel. A massively parallel tensor contraction framework for coupled-cluster computations. Journal of Parallel and Distributed Computing, 74(12):3176-3190, Dec. 2014.

[47] S. Toledo. A survey of out-of-core algorithms in numerical linear algebra. In External Memory Algorithms and Visualization, pages 161-180. American Mathematical Society Press, 1999.

[48] Top500. Top 500 Supercomputer Sites, June 2019. https://www. top500.org/lists/2019/06/.

[49] S. J. Treichler. Realm: Performance Portability through Composable Asynchrony. PhD thesis, Stanford University, 2014.

[50] R. A. Van De Geijn and J. Watts. SUMMA: scalable universal matrix multiplication algorithm. Concurrency: Practice and Experience, 9(4):255-274, 1997. 


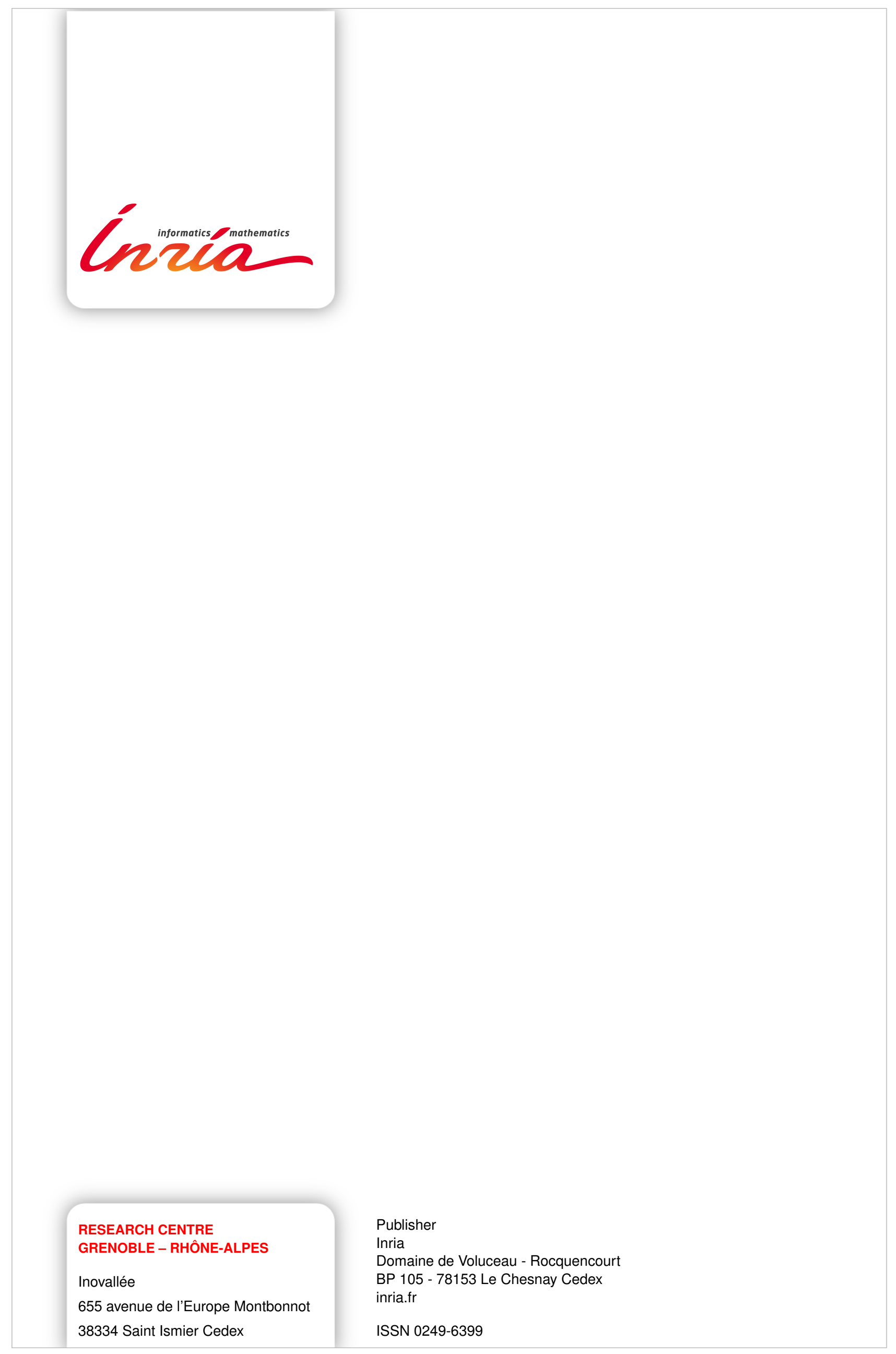

